\title{
Feasibility of Serverless Cloud Services for Disaster Management Information Systems
}

\author{
Tayyaba Asghar \\ University of Lahore \\ University of Gujrat \\ Gujrat, Pakistan \\ tayyaba.asghar@uog.edu.pk \\ Zia ul Qayyum \\ University of Gujrat \\ Gujrat, Pakistan \\ Ziaqayyum@gmail.com
}

\author{
Saqib Rasool \\ Information Technology University \\ University of Gujrat \\ Gujrat, Pakistan \\ saqib@ieee.io
}

\author{
Adnan Noor Mian \\ Information Technology University \\ Lahore, Pakistan \\ adnan.noor@itu.edu.pk
}

\author{
Muddesar Iqbal \\ School of Engineering \\ London South Bank University \\ London, UK \\ m.iqbal@1sbu.ac.uk \\ George Ubakanma \\ London South Bank University \\ London, UK \\ ubakang@lsbu.ac.uk
}

\begin{abstract}
Serverless is the new generation of cloud services that supports the pay-per-use policy in true spirit by charging only for the execution time of the hosted code. Amazon introduced serverless service of Lambda in 2014 and it is consider as the most popular serverless cloud service till date. This paper focuses on the serverless cloud services of Lambda and elaborates the importance of Lambda based serverless cloud services for hosting the disaster management information systems (DMIS). We have identified two repeatedly occurring phases of the life cycle of a DMIS viz. low activity phase and high activity phase. Our findings state that serverless cloud services are well-suited for both of these phases of a DMIS. Serverless reduces the operational cost during the low activity phase by detaching the code from running containers and it improves the scalability during the high activity phase by quickly assigning the already available containers from the container pool. However, this all comes with the price of reduced QoS (Quality of Service) for initial requests after specific idle duration and our experimental results report the QoS degradation with respect to idle time for Lambda service.
\end{abstract}

Keywords-FaaS; Serverless; Lambda; QoS; DMIS; Disaster;

\section{INTRODUCTION}

Serverless cloud services offer the fine-grained pay-peruse access to auto-scaling cloud resources and is achieved by applying no charge policy for idle applications [1]. Serverless services are also known as FaaS (Function as a Service) [2] as each service corresponds to a stateless (often light-weight) function. Each of these functions are inherently capable of quickly scaling against the bursty traffic and this scalability is entirely achieved by the cloud vendor without requiring any scalability knowledge by the user hosting that functions. Lambda [3] is the most popular FaaS and it was launched by Amazon in late 2014. Although Amazon deploys the serverless Lambda functions through containers but these are more robust and scalable [4] as compare to the Elastic BeanStalk, a server based container service [4].
V. Cardellini et. al [5] has identified the missing support of efficient resource allocation by cloud service providers for reducing the cost and ensuring the auto-scalability against two types of traffic viz. 1) bursty and 2) unpredictable. S. Hendrickson et al. [4] have proved that Lambda has solved the first problem of efficient auto-scaling against the bursty traffic by sharing a pool of containers among multiple instances of different applications. However, Lambda is not much effective for the unpredicted traffic.

J. Weinman [1] has identified the limitation of Lambda to handle the second problem as it is not capable of maintaining Quality of Service (QoS) for the initials requests after an idle time duration. It is also suggested to evaluate the performance of Lambda against the personalized requirements, before using it in production. Reason for reduced QoS is cold start [6] which removes the idle functions and restores back these after the next request arrives. Although it ensures the optimal utilization of resources but it also reduces the QoS for the initial requests sent after a specific idle time. More details of cold start are covered in section three.

Disaster Management Information System (DMIS) helps in the better execution of the assistance operations during and just after the disaster. It also helps in applying the lessons learned from previous disasters to improve the relief operations [7]. However, DMIS mostly remains idle during the time between two disasters and it suddenly generates the bursty traffic during the rehabilitation process. We have termed both of these phases as low and high activity phases respectively. In this paper, we have presented that serverless can support both of these phases with a QoS tradoff for few initial requests. Section two elaborates the evolution of virtualization with respect to optimal resource utilization. Section three explains the effectiveness of serverless for low and high activity phases of DMIS. Section four enlists related work and last section concludes the paper. 
(A)

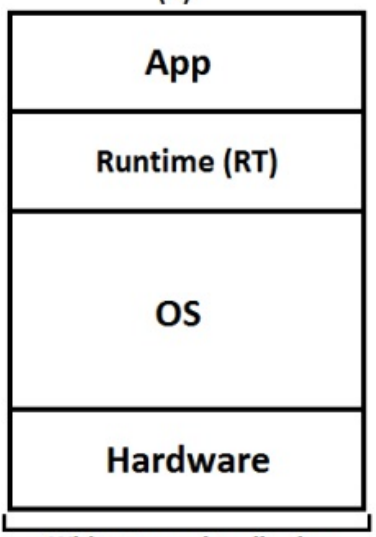

Without any virtualization
(B)

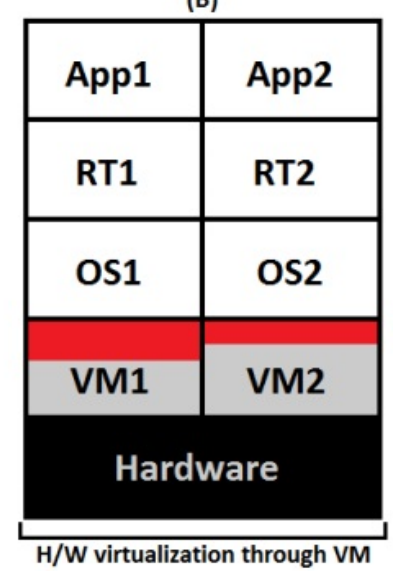

(c)

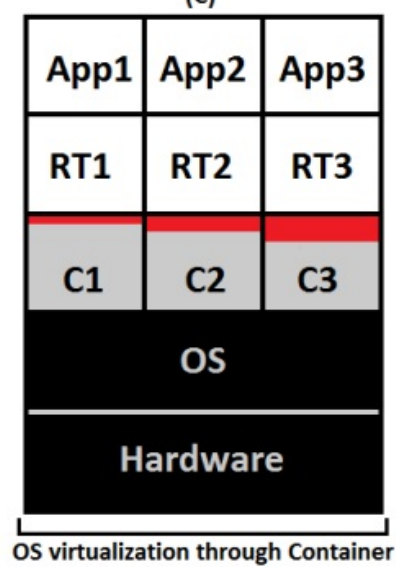

(D)

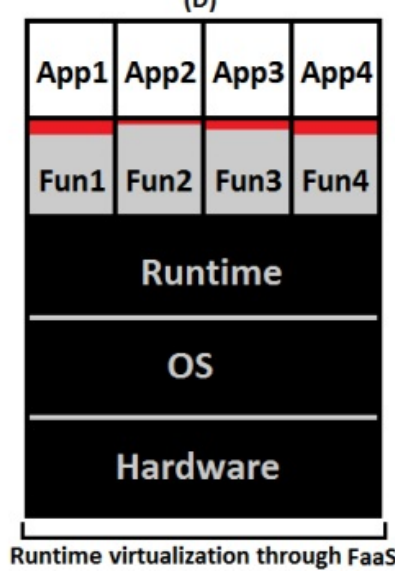

Figure 1. Evolution of virtualization from bare-metal deployments to three different virtualization models of cloud services

\section{Evolution of Virtualization}

In early days, the computation was tightly bound to hardware and applications ran directly on hardware machines. It not only resulted in wastage of system resources but also confined applications to vertical scalability [8] which is achieved by increasing the capacity of an individual machine. Bare-metal deployments also make it difficult to horizontally scale [9] the applications which is achieved by distributing an application over multiple machines to share the load among these.

Attaining the horizontal scalability through physical hardware results in two main challenges of 1) quickly launching new machines for handling the busty traffic and and 2) handling the sudden and unexpected hardware failures of physical machines. Virtualization offers the solution to both of these problems by efficiently managing and distributing the physical resources and also by imposing different application development practices for simplifying the horizontal scalability of deployed applications and this led to the beginning of cloud era.

Fig. 1 contains four parts where part A is showing a baremetal server without any virtualization while other three sections represent three major virtualization phases in the history of cloud computing with respect to the optimization of cloud resources. There are four different colors used in Fig. 1 and each of these are listed below:

Background color of Black represents the virtualizaiton which is controlled by the cloud service provider.

Background color of Grey represents the platforms that are used by the developers for hosting their codes on virtualized cloud infrastructure.

Background color of Red represents the wasted resources of the hosting platforms.

Background color of White represents the resources that are purely controlled by the developers that are using cloud services hosting their applications.

\section{A. Hardware virtualization through VM}

VMs (Virtual Machines) offer the hardware virtualization which enables the sharing of a physical machine among multiple applications. However, it does not ensure the efficient resource consumption during the low activity phase of a DMIS. This is because each VM has its own OS (Operating System) and it holds the computational resources; even if the application is in idle phase. Moreover, if applications are in running phase, it is not very often that these will be utilizing all the designated resources of a VM. Red color in part B of Fig. 1 represents the resources that are wasted due to the under-utilization by the VM.

\section{B. OS virtualization through Container}

Containers ensure the OS virtualization by sharing same kernel for offering multiple runtimes. Each runtime is further designated for an individual application. Containers are amongst the light-weight virtualization options with relatively less startup time as compare to the VMs. However, multiple containers can share only a single type of OSkernel [10]. Part C of Fig. 1 shows that more containers can be deployed with relatively less resources as compare to VM. Moreover resource wastage is also less in containers as compare to VMs which can also be interpreted from the difference of red color in both part B and C of Fig. 1.

\section{Runtime virtualization through FaaS (Serverless)}

Both VM and containers do not impose application development patterns for achieving the horizontal scalability. However, runtime virtualization imposes the application developers to divide the application functionality into finegrained, light-weight and stateless functions and deploy each of these functions as an independent service and therefore, it is also known as Function-as-a-Service. It not only ensures the maximum resource sharing but also improves the consumption of allocated resources and same is depicted from less amount of red color in part D of Fig. 1. 


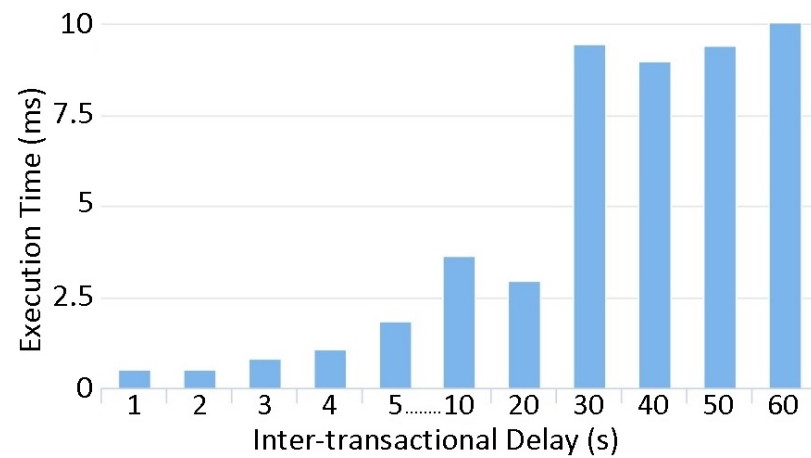

Figure 2. QoS degradation of a Lambda service against the time-varying workloads

\section{FeASibility OF SERVERLESS FOR DMIS}

Life time of a DMIS can be divided into two distinct phases of 1) low activity phase during the time duration between two disasters and 2) high activity phase during the relief operation of a disaster. This section explains the low and high activity phases of a DMIS with respect to three virtualization models discussed in last section and also elaborates the reason for reduced QoS due to the cold start in serverless deployments.

\section{A. Low activity phase}

Information systems for disaster management generates bursty traffic during the disaster but remain almost idle for the time period between two disasters. Therefore, if a DMIS is hosted at VM or container based virtualized infrastructure then it will waste the resources during the idle time. However, in case of deployment on a serverless infrastructure, it will result in the reduction of operational cost due to cold start.

\section{B. High activity phase}

DMIS generates huge traffic during the relief operations after a disaster. If a DMIS is hosted on a VM or a container it will take time to launch new VM and containers and it reduces the overall QoS. This reduction in QoS will be experienced during the launch of every new instance due to the increased traffic on existing instances. Hence, QoS will not remain consistent throughout the relief operations. However, when same DMIS is hosted on serverless infrastructure, it will initially experience the degradation in QoS due to the cold start. However, after the first few requests, QoS will remain consistent due to the scalable nature of the serverless cloud services. In case of serverless deployments, no time is wasted for launching new containers or VMs because a pool of containers is already maintained and just the allocation of code to a container needs to be done for scaling to support the bursty traffic of an application hosted on serverless.

\section{Reduced QoS in serverless due to cold start}

QoS and SLA (Service Level Agreement) guarantees are considered amongst the major challenges of cloud services and these are more crucial for FaaS due to the ad-hoc assignment of containers to serverless functions. This autonomous process of resource management and assignment degrades the QoS for initial requests as it is focused on reducing the operational cost of cloud vendors. A container pool is maintained by the Amazon and a Lambda function is assigned to one container upon the request. After the completion of function execution, container is released back to the container pool, if no other execution request is arrived for the some time.

Ad-hoc algorithm of resource management tries to assign the same container to the function on any future requests. However, there is no guarantee that same container is assigned again for the same request and it further increases the response time. This increase in response time results in further degradation in QoS because both QoS and execution time are inversely proportional.

We have performed extensive experiments for finding the QoS degradation in Lambda Service of Amazon by sending 1100 requests in two sets of different ranges of inter-transactional delay. First set contains 500 time-varying requests with inter-transactional delays of 1, 2, 3, 4 and 5 seconds each and second set contains 600 requests for intertransactional delays of 10, 20, 30, 40, 50 and 60 each. Average of 100 requests against each of the 11 different intertransactional delays is presented in Fig 2. It can be deducted from the presented results that the inter-transactional delay is the prominent factor for deciding the container detaching. For lesser inter-transactional delays, there were few chances of container detaching and it resulted in less average execution time. However, once a container is switched and function is assigned to a new container, it increases the execution time which resulted in QoS degradation.

Results presented in Fig. 2 were collected in June 2017 and were based on 1100 non-overlapping requests to a Lambda function. However, in case of overlapping requests, almost all initial request experience the similar degradation in QoS and this can further reduce the average QoS values presented in Fig. 2. Moreover, memory size of hosted function also effects the invocation time of a function. Detaching of code from the containers results in cold start and this problem can be solved by warming the serverless functions through periodic invocation of the deployed functions.

\section{LITERATURE REVIEW}

In this paper, we have focused on finding the feasibility of serverless cloud services for hosting the DMIS. Serverless cloud services has already been used for hosting few functionalities during the relief operation of a disaster. However, it is not proposed for hosting DMIS by identifying its support for both low and high activity phases of a DMIS. 
Serverless cloud services are used by a system for disaster managers, known as CongiCity [11]. However, this whole system was not hosted on the serverless and they deployed just a single reporting module on serverless. This reporting module uses the real-time social media streams from twitter, facebook, telegram and used these for flood mapping in Chennai, India. This flood mapping through social media streams helped in the efficient decision making during the flood. This study has used the Lambda service of Amazon for deploying the real-time reporting module from social media accounts while we have proposed the deployment of whole DMIS on serverless cloud service.

Serverless has also been used for reducing the reunification time of children during the disaster scenarios [12]. This is achieved by running an image processing algorithm over the pictures of the separated children for finding their legal parents. This whole module was deployed on an opensource serverless platform by IBM, known as OpenWhisk [13]. Serverless deployment of this module reduces the reunification time of children with their legal parents due to high scalability of serverless cloud services. They have also deployed only a single module over serverless while we have proposed the deployment of whole DMIS on serverless cloud services. We have not only presented serverless for hosting DMIS but also reported the QoS degradation due to cold start in serverless.

\section{CONCLUSION}

We have identified two repeatedly occurring phases of a DMIS life cycle. First phase is the high activity phase which occurs during the relief operations of a disaster and second is the low activity phase which is based on the duration between two disasters. Serverless is good for efficient resource management by removing the running code of DMIS during the low activity phase for reducing the operational cost. It also gives more scalability during the high activity phase of DMIS by using an already maintained pool of containers.

This paper has identified the strength of serverless for supporting both low and high activity phases. We have also evaluated one of the limitation of serverless, known as cold start, which results in the reduced QoS for initial request after an idle time duration. We have performed experiments on Lambda, which is the most popular serverless cloud service by Amazon and we have reported the extent to which QoS is degraded with respect to idle time in Lambda service of Amazon.

\section{ACKNOWLEDGMENT}

The authors are grateful for the support received from the transnational CiProVoT (Civil Protection Volunteers Training) project. The project is co-funded by ERAMUS+ programme of the European Union.

\section{REFERENCES}

[1] A. Eivy, "Be wary of the economics of" serverless" cloud computing," IEEE Cloud Computing, vol. 4, no. 2, pp. 6-12, 2017.

[2] J. Spillner, "Snafu: Function-as-a-service (faas) runtime design and implementation," arXiv preprint arXiv:1703.07562, 2017.

[3] G. C. Fox, V. Ishakian, V. Muthusamy, and A. Slominski, "Status of serverless computing and function-as-aservice (faas) in industry and research," arXiv preprint arXiv:1708.08028, 2017.

[4] S. Hendrickson, S. Sturdevant, T. Harter, V. Venkataramani, A. C. Arpaci-Dusseau, and R. H. Arpaci-Dusseau, "Serverless computation with openlambda," Elastic, vol. 60, p. 80, 2016.

[5] V. Cardellini, E. Casalicchio, and L. Silvestri, "Service level provisioning for cloud-based applications," in Grid and Cloud Computing: Concepts, Methodologies, Tools and Applications. IGI Global, 2012, pp. 1479-1500.

[6] M. Stigler, "Understanding serverless computing," in Beginning Serverless Computing. Springer, 2018, pp. 1-14.

[7] J. Lee and T. Bui, "A template-based methodology for disaster management information systems," in System Sciences, 2000. Proceedings of the 33rd Annual Hawaii International Conference on. IEEE, 2000, pp. 7-pp.

[8] L. Mei, W. K. Chan, and T. Tse, "A tale of clouds: Paradigm comparisons and some thoughts on research issues," in AsiaPacific Services Computing Conference, 2008. APSCC'08. IEEE. Ieee, 2008, pp. 464-469.

[9] B. P. Rimal, E. Choi, and I. Lumb, "A taxonomy and survey of cloud computing systems," in INC, IMS and IDC, 2009. NCM'09. Fifth International Joint Conference on. Ieee, 2009, pp. $44-51$.

[10] P. Andreetto, J. Astalos, M. Dobrucky, A. Giachetti, D. Rebatto, A. Rosato, V. Tran, M. Verlato, and L. Zangrando, "Egi federated platforms supporting accelerated computing."

[11] D. Sridhar and M. Priyaa, "Real-time flood mapping for disaster management decision support in chennai," Ph.D. dissertation, Massachusetts Institute of Technology, 2017.

[12] K. Coleman, F. Esposito, and R. Charney, "Speeding up children reunification in disaster scenarios via serverless computing," in Proceedings of the 2nd International Workshop on Serverless Computing. ACM, 2017, pp. 5-5.

[13] N. Bila, P. Dettori, A. Kanso, Y. Watanabe, and A. Youssef, "Leveraging the serverless architecture for securing linux containers," in Distributed Computing Systems Workshops (ICDCSW), 2017 IEEE 37th International Conference on. IEEE, 2017, pp. 401-404. 